\title{
Las preguntas que el clínico debe hacerse en un paciente hipertenso al sospechar una Hipertensión Secundaria
}

\author{
Drs. Rodrigo Tagle V. ${ }^{1}$ y Mónica Acevedo B. ${ }^{2}$ \\ Departamentos de Nefrología1 y de Enfermedades Cardiovasculares ${ }^{2}$. \\ Pontificia Universidad Católica de Chile \\ Recibido el 1 de junio de 2009. Aceptado el 15 de junio de 2009
}

Rev Chil Cardiol 2009; 28: 214-221

Los pacientes con Hipertensión Arterial (HTA) pueden clasificarse según si éstos presentan o no una etiología clara y evidente $y$, si tienen o no mecanismos hipertensógenos conocidos. Así, a los primeros se les clasifica como HTA secundaria (HTAS), y a aquellos que no presentan una causa determinada, se les clasifica como HTA esencial o primaria1.

Existe aún en la actualidad la idea de que es razonable la búsqueda de una HTAS, ya que su diagnóstico y posterior tratamiento "especifico" llevará al paciente a la normotensión, definida como una presión arterial (PA) $<140 \mathrm{mmHg}$ de sistólica y $<90 \mathrm{mmHg}$ de diastólica, sin la utilización de drogas antihipertensivas, logrando "curación" de su enfermedad. Sin embargo, los diferentes estudios, particularmente de series clínicas, no concuerdan con esa idea. Así se justifica analizar cuándo se debe buscar una HTAS, considerando desde su frecuencia y características clínicas, hasta los resultados de las terapias de las diversas entidades que pueden causar una HTAS con sus mecanismos. Más aún si el estudio etiológico de la HTA tiene costos, que van desde el valor de los exámenes hasta sus riesgos, pasando por sus molestias y tiempo utilizado. Esta revisión pretende entonces, proporcionar al clínico conceptos clínicos actualizados sobre la HTAS, sus agentes causales y posibilidades de curación. La primera pregunta que debe hacerse el clínico cuando se plantea la posibilidad de una HTAS es el por qué debemos buscarla. Las razones de su búsqueda son tres 2 : a) El control de la HTA en un paciente con HTAS implica, habitualmente, un tratamiento médico o quirúrgico específico.

b) En algunos pacientes con HTAS puede lograrse la corrección completa o parcial de la HTA.

c) La HTAS puede ser una manifestación de alguna neoplasia hormonalmente activa y eventualmente maligna, como un feocromocitoma.

La segunda pregunta se refiere a conocer qué enfermedades hipertensivas tienen un tratamiento específico. Las enfermedades hipertensivas que son tratables $\mathrm{y}$, eventualmente corregibles por métodos quirúrgicos y/o intervencionales son: a) estenosis de la arteria renal, b) nefropatía unilateral secundaria a una hidronefrosis o pielonefritis crónica, c) adenomas productores de aldosterona, d) síndrome de Cushing, e) coartación de la aorta, f) feocromocitoma, g) hiperparatiroidismo primario, y h) acromegalia. En cambio, aquellas que tienen un tratamiento médico específico y son potencialmente corregibles son: a) hiperaldosteronismo por hiperplasia suprarrenal, b) hiperaldosteronismo supresible por dexametasona, c) hipertiroidismo, d) hipotiroidismo, y e) síndrome de apnea del sueño (SAOS).

Entre estas entidades merecen especial mención, los dos últimos, el hipotiroidismo y el SAOS, por su significativa prevalencia en la población general. El hipotiroidismo es una causa ampliamente aceptada de HTA, pero habitualmente subvalorada. La HTA es tres veces más frecuente en los sujetos

Correspondencia: Dr. Rodrigo Tagle $\mathrm{V}$

Departamentos de Nefrología

Pontificia Universidad Católica de Chile

Correo Electrónico: rtagle@med.puc.cl 
hipotiroideos comparados con aquellos eutiroideos. Esta entidad, con sus múltiples cambios metabólicos sistémicos puede además modificar y/o enmascarar otras formas de HTAS, como por ejemplo, la hiporreninemia ocasionada por la disminución del efecto de la tiroxina sobre la secreción de renina. Típicamente, la HTA inducida por el hipotiroidismo es una HTA de tipo diastólica aislada, por un aumento de la resistencia vascular periférica con un débito cardiaco y volemia disminuidos. El aumento del tono vascular se debe a: la ausencia del efecto vasodilatador de la hormona T3, niveles plasmáticos elevados de catecolaminas y a una disminución de los receptores vasculares beta- 2 adrenérgicos ${ }^{1}$.

EL SAOS constituye un factor de riesgo para HTA, independiente de la presencia de obesidad, de la edad o el sexo. El riesgo de presentar HTA se incrementa de modo casi lineal cuando se considera el número de apneas/hipopneas por hora de sueño. Los mecanismos involucrados se relacionan a un aumento del tono simpático inducidos por la hipoxemia y por los repetidos episodios de alteración del sueño, fenómenos llamados "microdespertares". Así se explica la pérdida inicial del descenso fisiológico nocturno de la PA (dip) y, finalmente la HTA diurna1,2.
La tercera pregunta está dirigida a saber cuál es la probabilidad de curación de la HTA en un paciente con HTAS. Es decir, en cuáles de las entidades anteriores, una vez que han sido tratadas, la HTA propiamente tal, puede ser curada y/o lograda la normotensión, definida como una presión arterial $<140 \mathrm{mmHg}$ de sistólica y $<90 \mathrm{mmHg}$ de diastólica, sin la utilización de drogas antihipertensivas.

En general, la frecuencia de normotensión, después de tratar algunas de estas enfermedades mediante terapias quirúrgicas y/o intervencionales, es menor a $50 \%$, dependiendo fundamentalmente de la entidad hipertensiva, de la edad del paciente y del tiempo transcurrido desde que el sujeto se ha mantenido hipertenso.

No todas las entidades que se presentan con HTA tienen similares tasas de curación. Así, por ejemplo, el $50 \%$ de los adultos operados de coartación de la aorta $(2,3), 50 \%$ de los pacientes operados de adenomas productores de aldosterona (Tabla $\mathrm{N}^{\circ}$ 1) (4), $8 \%$ a $22 \%$ de los pacientes con estenosis de las arterias renales 5 , dependiendo de la enfermedad causal (Figura 1) y, alrededor del $90 \%$ de los pacientes operados de feocromocitoma 6 logran curación de su HTA.

Tabla 1: Tasas de Curación de la HTA en adenomas productores de aldosterona operados.

\begin{tabular}{llccc}
\hline Autor & Año & Curación: $\mathbf{N}^{\circ}$ & - & \% Pacientes \\
\hline Pagny, & 1987 & 28 & 47.5 \\
Favia, & 1992 & 35 & 70.0 \\
Obara, & 1992 & 39 & 61.9 \\
Stowasser, & 1994 & 30 & 54.5 \\
Proye, & 1998 & 56 & 56.0 \\
Sawka, & 2001 & 31 & 33.3 \\
Total & 219 & 52.1 & \\
\hline
\end{tabular}

${ }^{*}$ Adaptado de referencia 4. 


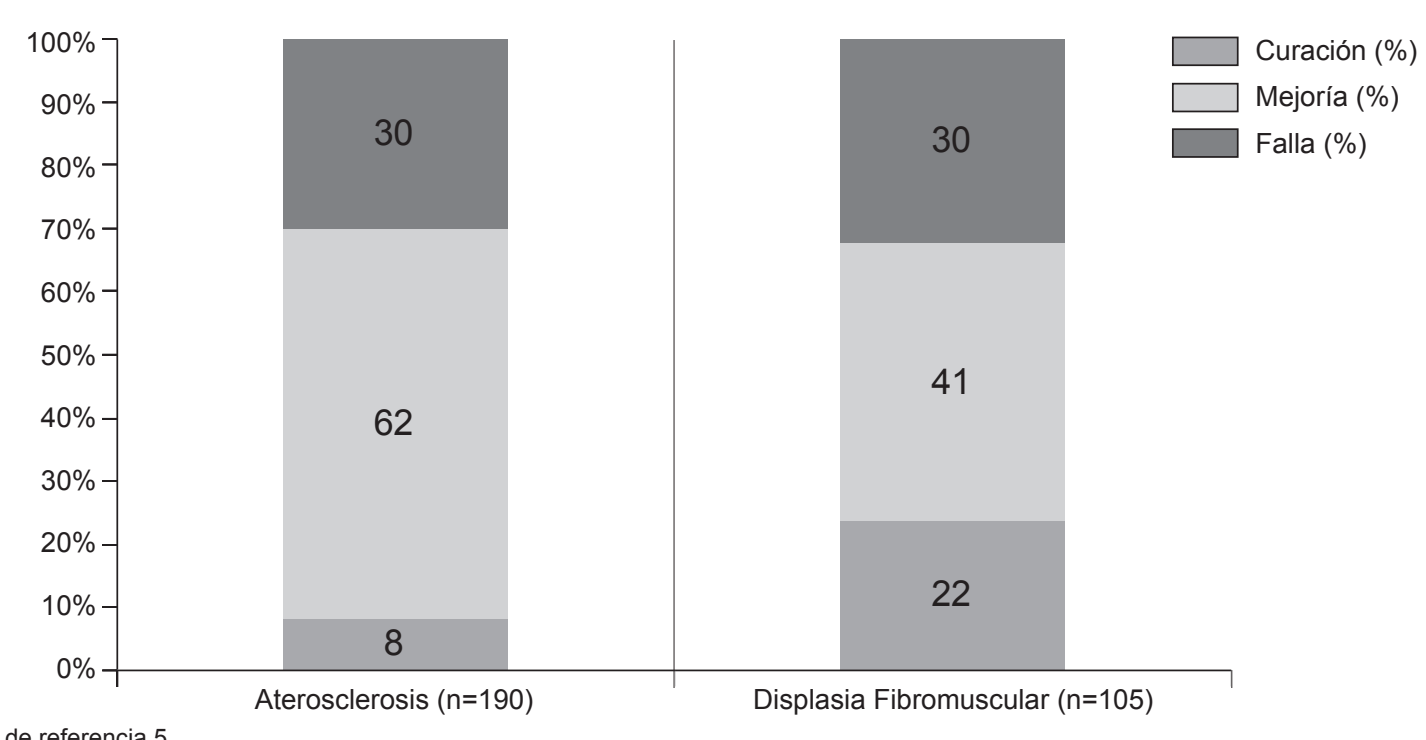

*Adaptado de referencia 5.

Figura 1: Efecto de la Angioplastia en Estenosis de Arterias Renales según causa.

La tasa de curación de la HTA en estas enfermedades, por otra parte, es claramente influida por la edad del paciente. A mayor edad, especialmente después de los 40 años, es más probable que se agregue una HTA esencial, cuya prevalencia en la población general es muy alta. Así, a mayor edad del paciente, menor es la tasa de normotensión post-procedimiento. En consecuencia, estas entidades tienen una mayor tasa de curación en sujetos jóvenes, como se observa en aquellos pacientes menores de 40 años afectados de estenosis de las arterias renales por displasias de las arterias renales 7 . Pero, la posibilidad de lograr la normotensión es significativamente menor con cualquier intervención vascular, quirúrgica y/o endovascular cuando la estenosis de las arterias renales se debe a aterosclerosis en sujetos mayores de 55 años $^{8}$. (Figura 2)

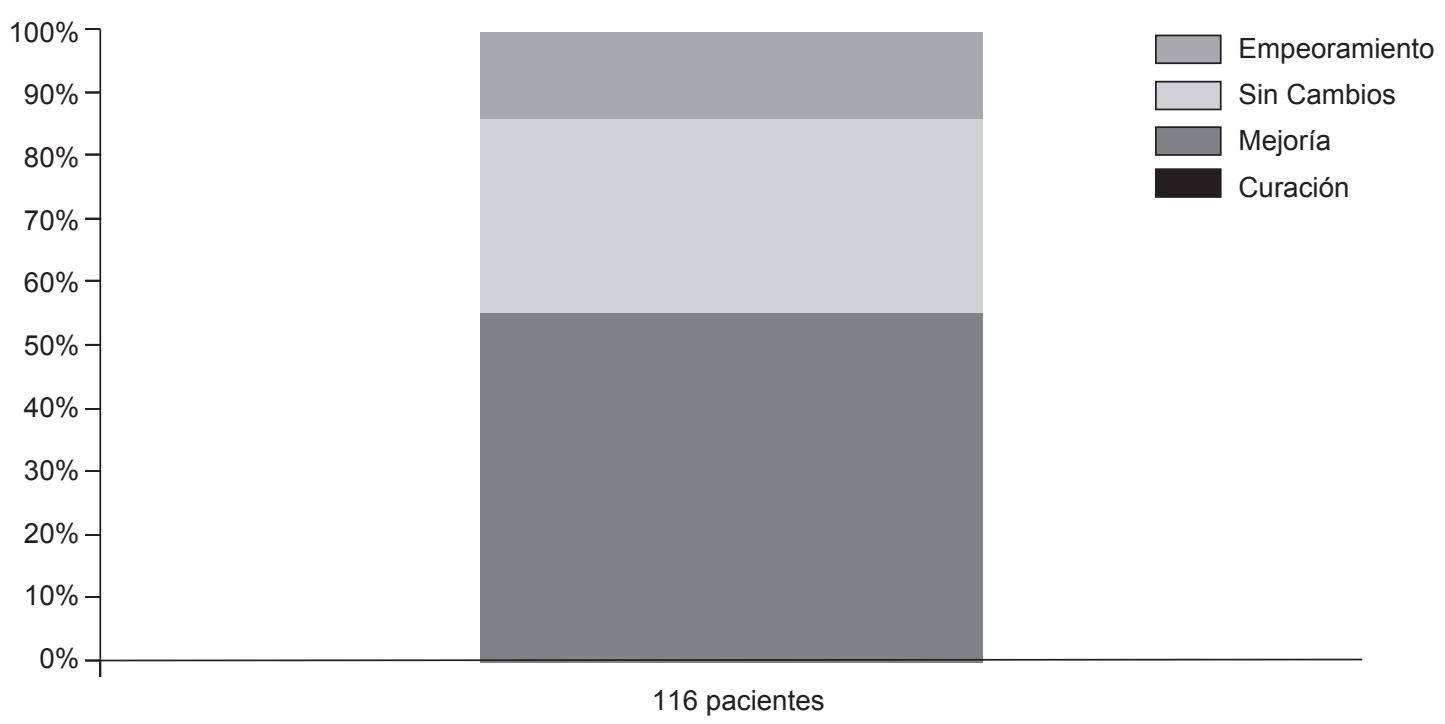

Figura 2: Efecto del stent en el control de la HTA en estenosis aterosclerotica de arterias renales *Adaptado de referencia 8 . 
Por último, la importancia del tiempo transcurrido con HTA en la tasa de curación de estas entidades se ha demostrado a través de diversos estudios fisiopatológicos y especialmente clínicos, particularmente para el adenoma suprarrenal productor de aldosterona y la estenosis renovascular. Estudios señeros sobre la fisiopatología de la HTA renovascular unilateral en el modelo de un clip y dos riñones (2K1C) detectaron el rol del tiempo en la posibilidad de curación de la HTA, dando origen al concepto de las fases de la HTA renovascular (Figura 3). En general, cuando el paciente tiene una historia de HTAS menor a 5 años de evolución, ésta se asocia a una alta tasa de curación. En cambio, en aquel paciente que ha tenido HTAS por más de 5 años se hace más difícil la curación, cualquiera sea la causa de la HTAS. Al parecer con los años se va produciendo una alteración no modificable de la morfología vascular arterial por fibrosis e hipertrofia, y una alteración de la curva presión - natriuresis por daño de la microcirculación intrarrenal.

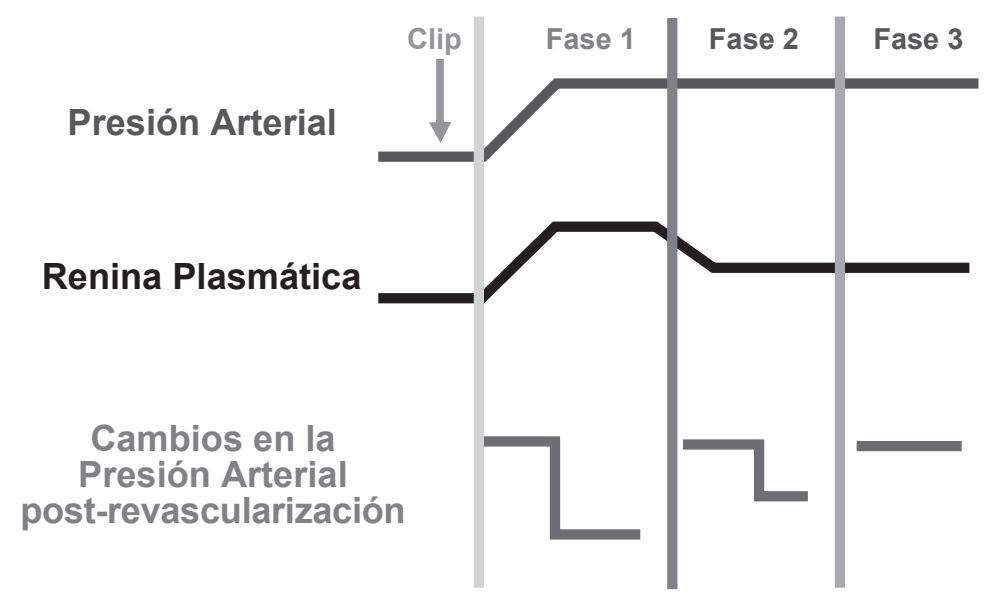

Figura 3: Fases de la Hipertensión Renovascular Unilateral

Respecto a aquellas HTAS que tienen un tratamiento médico específico (hipertiroidismo, hipotiroidismo, SAOS, etc) y que, supuestamente serian corregibles con el tratamiento dirigido a la enfermedad causal, tampoco se logra en un $100 \%$ la normotensión. En el hipotiroidismo, se logra la normotensión con la terapia sustitutiva de T4 sólo en un $33 \%$ a un $50 \%$ de los pacientes 1 . En la serie del Dr. Anderson, analizada posteriormente, en donde se atribuyó el hipotiroidismo como causa de HTAS (3\% de 4429 pacientes), sólo un tercio de ellos se normotensaron con la terapia sustitutiva.

En una entidad más reciente como el SAOS, en los diferentes metanálisis se observa que los resultados de la terapia con CPAP nasal son disímiles, aunque efectivamente muestran una tendencia a mejorar el control de la PA, pero rara vez se logra la suspensión completa de los antihipertensivos. Sí destaca el hecho de que su rendimiento es mayor en aquellos con HTA refractaria y en aquellos con más episodios de apneas 1,2 .

La HTAS que sí son curables y habitualmente subvaloradas, son las HTAS a drogas y la relacionada a la obesidad.

Habitualmente, en la HTAS a drogas cuando se suspende el fármaco y/o agente, la PA retorna a valores normales. HTA secundaria a drogas, aunque es una afección poco frecuente en general, menor de un $1 \%$ entre los diagnósticos de HTAS, pero que, dependiendo del fármaco y del estado de la función renal previa del paciente, puede llegar a ser frecuente y/o severa. Entre las drogas más frecuentemente relacionadas con elevación de la PA se encuentran: antiinflamatorios no esteroidales tradicionales (AINEs), inhibidores de la COX-2 (iCOX2), descongestionantes, antidepresivos tricíclicos, drogas antijaquecosas, inhibidores de la MAO, corticoides, anabólicos, anticonceptivos orales con 30 a 35 ug de estrógenos (ACO), ciclosporina, 
tacrolimus, cocaína y los medicamentos utilizados para bajar de peso (anorexígenos) tales como: sibutramina, phentermine y anfetaminas ${ }^{9}$. Entre las drogas habitualmente no mencionadas se encuentran los fármacos que son antagonistas de los receptores dopaminérgicos, ya sean con efectos centrales (drogas antiparkinsonianas como bromocriptina) y/o periféricos, tales como metoclopramida, domperidona, sulpiride o veralaprida, que pueden, al bloquear los receptores dopaminérgicos tipo DA-2 pre-sinápticos, originar un aumento de la liberación de noradrenalina de las terminales nerviosas centrales y periféricas. Normalmente, estos receptores DA-2 pre-sinápticos actúan de manera semejante a los receptores alfa-2 adrenérgicos pre-sinápticos, disminuyendo la liberación exocítica de noradrenalina. El bloqueo de estos receptores dopaminérgicos pre-sinápticos permite comprender entonces la fisiopatología de este tipo de HTAS. A continuación se enumeran los mecanismos involucrados en la generación de HTA por fármacos con sus representantes más típicos. En general, puede aparecer un mecanismo patogénico único o una combinación de todos o algunos de ellos:

1. Expansión de volumen a través de la retención de agua y sal (Ej.: AINEs, iCOX2)

2. Aumento de la actividad adrenérgica

(Ej.: anorexígenos, antidepresivos)

3. Activación del SRAA (Ej.: ACO).

4. Falta de efectividad de hormonas vasodilatadores (Ej.: corticoides, ciclosporina).

Un agente, no siempre considerado, es el alcohol, cuyo exceso (más de tres tragos diarios) también puede causar HTAS. Uno de los mecanismos involucrados más importantes en la generación de HTA en estos sujetos es la activación del sistema nervioso simpático central10.

Entre los "nuevos" agentes hipertensógenos se encuentran tanto drogas como hierbas, como por ejemplo: marihuana, sanguinaria, Ma huang (supresor del apetito), lopinavir (antiretroviral), eritropoyetina y el ginseng11. Estos dos últimos comparten una vía hipertensógena común, ya que ambos bloquean la biodisponibilidad del oxido nítrico9,11.

No podemos dejar de mencionar en esta categoría al licorice (hierba Glycyrrhiza Glabra), usado como edulcorante en algunos países, que inhibe la enzima $11 ß$ hidroxiesteroide dehidrogenasa tipo 2, facilitando la activación del receptor minerolocorticoideo por el cortisol, ocasionando así un subtipo de Hiperaldosteronismo primario.

Aunque la nicotina (cigarrillos, puros y cápsulas masticables para dejar de fumar) y cafeína son señalados como agentes hipertensógenos, éstos sólo producen alzas transitorias de la PA, y en consecuencia, no son causa de HTAS. Pero estos agentes, sí realzan la importancia de no haber ingerido cafeína ni haber fumado en los 30 minutos previos a la medición de la PA.

Por otro lado, la obesidad puede causar HTAS12. Los mecanismos involucrados en esta forma de HTAS son variados e incluyen entre otros: activación del sistema simpático central, aumento de la retención de sodio y agua mediada por la hiperinsulinemia, aumentos de los niveles de angiotensina II, y aumento de los niveles de mineralocorticoides ${ }^{12}$. La reducción significativa y sostenida del peso logra la normotensión en un $50 \%$ de estos pacientes (9). Recientemente, esto está siendo corroborado con las series clínicas de pacientes obesos e hipertensos sometidos a cirugía bariátrica, como fue publicado en nuestro país (13). Finalmente en esta pregunta, debemos señalar que existen ciertas causas de HTAS que claramente no son corregibles como son las enfermedades crónicas del parénquima renal, entre las cuales se encuentran la enfermedad poliquística renal del adulto y las glomerulonefritis crónicas.

Puede concluirse entonces que, aunque existen ciertas formas curables, no toda HTAS es curable.

La cuarta pregunta corresponde a conocer cuál es la real prevalencia de HTAS. Desgraciadamente, aún no existe una respuesta definitiva. Se acepta que a nivel de atención terciaria o especializada puede ser de hasta un $30 \%$. A nivel de atención primaria, sin embargo, existe cierto consenso de una prevalencia alrededor de $5 \% 14,15,16$.

No existen estudios en Chile sobre la frecuencia de la HTAS. Sobre este respecto, debemos mencionar que en un estudio sobre hiperaldosteronismo primario en atención primaria, la prevalencia de éste fue $6,1 \%$. La población analizada eran hipertensos catalogados 
como esenciales, o sea, no incluía aquellos con síntomas, signos y/o exámenes de laboratorio sugerentes de HTAS, ya que habitualmente son derivados precozmente al nivel terciario 17.

En el contexto de la última encuesta nacional de salud, año 2003, en Chile existiría una población estimada de 1.400 .000 hipertensos, de los cuales sólo 70.000 (5\%) personas tendrían una HTAS. De estos datos se deduce que si todos los hipertensos fueran estudiados en forma "extensa y completa", se harían a lo menos 1.330 .000 estudios innecesarios, costosos y a veces peligrosos para encontrar sólo 70.000 personas con una enfermedad tratable etiológicamente.

En los escasos estudios, no más de 10 , sobre prevalencia y causas de HTAS (Figura 4), la prevalencia de HTAS fluctuó entre un $4,6 \%$ a un $10,9 \%$, con un promedio de $8,3 \pm 2,6 \% 14,18,19,20,21$.

Sí es necesario establecer que la prevalencia de la HTAS y de las diversas entidades que la pueden originar, pudiesen variar en el tiempo por el advenimiento de nuevas técnicas diagnósticas.

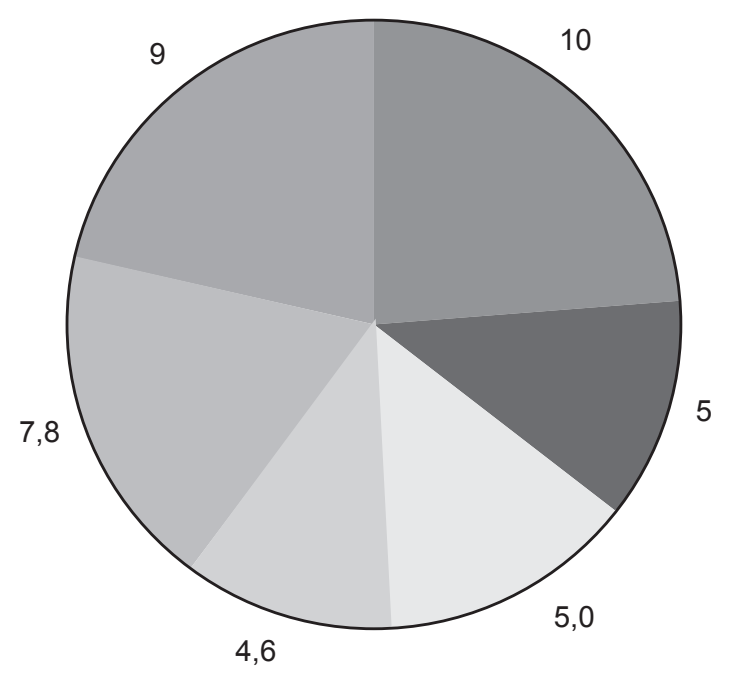

Gifford 1959

Berglund 1976

Rudnick 1977

Danielson 1981

Sinclair 1997

Omura 2001

Figura 4: Frecuencia de HTAS en diversos estudios.

La quinta pregunta se refiere a conocer cuáles son las características de la HTAS curable. Esto permitiría separar la población de hipertensos con mayores posibilidades de tener una HTAS curable, en otras palabras altas probabilidades pre test. Una buena historia clínica, examen físico completo y laboratorio general entregan ciertas claves clínicas, que indican qué enfermos tienen alta probabilidad pre test de tener una HTAS curable. En orden de prioridad son 15 :

- Hipertensos menores de 30 años. Especialmente aquellos sin historia familiar de hipertensión arterial.

- Hipertensos de reciente comienzo. Un paciente que se controlaba anualmente la PA y que siempre tenía valores de $120 / 80 \mathrm{mmHg}$, y ahora tiene valores de PA de 150/90 mmHg, constituye un excelente ejemplo.
- Hipertensos que presentan concomitantemente: cefalea, palpitaciones y sudoración. Su probabilidad pre test para feocromocitoma es de un $90 \% 16$, con una altísima tasa de normotensión post cirugía, cercana al $90 \% 6$.

- Hipertensos de genero femenino, menores de 40 años con soplo sistodiastólico en los flancos, orienta fuertemente a una fibrodisplasia de la arteria renal, con una tasa de normotensión post-intervención cercana al $22 \% 5$.

- Hipertensos resistentes: aquellos que no responden a 3 o más fármacos, de diferentes mecanismos antihipertensivos, en las dosis adecuadas, siendo uno de ellos un diurético.

La sexta pregunta se refiere al porqué deberíamos 
buscar HTAS en los pacientes con HTA resistente. La HTA resistente, definida como la persistencia de la HTA a pesar del tratamiento farmacológico en dosis plenas con tres o más drogas, de las cuales uno es un diurético, puede llegar a ser de fácil manejo y quizás curable. Esto ocurrirá si se bloquea el mecanismo generador de la HTAS. Ejemplo: en un hipertenso diagnosticado como esencial, pero que en realidad tiene de base un hiperaldosteronismo primario, se le prescribe inicialmente un diurético de tipo tiazídico; obviamente, el sujeto seguirá con PA elevadas; a este mismo sujeto, se le agrega un inhibidor de la enzima convertidora de angiotensina II, no produciéndose mayor cambio, como también, no se logra el objetivo de normotensión al agregarse un tercer fármaco, un bloqueador de los canales de calcio; finalmente, al mismo sujeto se le prescribe espironolactona, un diurético con efecto antimineralocorticoide; luego de 6 a 8 semanas ocurre una normalización de la PA. Este es un ejemplo de HTA de difícil manejo, en el cual, una HTA resistente, se normaliza al agregar el fármaco que actúa sobre el principal mecanismo hipertensógeno. Excelentes ejemplos de esta situación clínica, la constituyen el feocromocitoma y el hiperaldosteronismo primario, ya que las pautas de tratamiento de la HTA no incluyen habitualmente la prescripción de antihipertensivos, que bloquean los mecanismos hipertensógenos principales de estas dos enfermedades.

En relación al hiperaldosteronismo primario, ha sido señalado desde hace años como una causa frecuente de HTA de difícil control o resistente, como también fue observado en Chile ${ }^{17}$. En aquellos casos de HTA resistente debidos a una adenoma productor de aldosterona, la tasa de normotensión en este contexto clínico ha sido de un $33 \% 22$.

Por último, la séptima pregunta se refiere a cuáles son las causas más frecuentes de HTAS. Desafortunadamente, existen muy pocos estudios orientados a contestar esta pregunta de manera convincente. Tradicionalmente, se ha citado el estudio del Dr. Anderson de New York, en el cual se estudiaron pacientes hipertensos con sospecha de HTAS. A todos ellos se les realizó: electrolitos plasmáticos, creatinina, hormonas tiroideas, actividad de renina plasmática, catecolaminas plasmáticas, cortisol y aldosterona plasmática pre y post infusión de suero fisiológico. Así encontraron un $10.3 \%$ de HTAS. Las principales causas fueron HTA renovascular e hipotiroidismo18. (Tabla 2).

Tabla 2: Frecuencia de Causas de HTAS en estudio Dr. Anderson.

\begin{tabular}{lc}
\hline Causas & Porcentaje \\
\hline Renovascular & $3.1 \%$ \\
Hipotiroidismo & $3.0 \%$ \\
Insuficiencia Renal & $1.8 \%$ \\
Hiperaldosteronismo & $1.4 \%$ \\
Cushing & $0.5 \%$ \\
Feocromocitoma & $0.3 \%$ \\
Total & $10.3 \%$ \\
\hline
\end{tabular}

Tabla 3: Frecuencia de HTAS en diferentes estudios

\begin{tabular}{lcccccc}
\hline & Gifford & Berglund & Rudnick & Danielson & Sinclair & Omura \\
\hline & 1969 & 1976 & 1977 & 1981 & 1987 & 2004 \\
N ${ }^{\circ}$ Pacientes & 4339 & 689 & 665 & 1000 & 3783 & 1020 \\
Nefropatía & $5 \%$ & $4 \%$ & $5 \%$ & $2.4 \%$ & $5.6 \%$ & - \\
Renovascular & $4 \%$ & $1 \%$ & $0.2 \%$ & $1.0 \%$ & $0.7 \%$ & $0.5 \%$ \\
Coartación Aórtica & $1 \%$ & $0.1 \%$ & $0.2 \%$ & - & - & - \\
Hiperaldosteronismo & $0.5 \%$ & $0.1 \%$ & - & $0.1 \%$ & $0.3 \%$ & $5.9 \%$ \\
Cushing & $0.2 \%$ & - & $0.2 \%$ & $0.1 \%$ & $0.1 \%$ & $2.0 \%$ \\
Feocromocitoma & $0.2 \%$ & - & - & $0.2 \%$ & $0.1 \%$ & $0.6 \%$ \\
Anticonceptivos & - & - & $0.2 \%$ & $0.8 \%$ & $1.0 \%$ & - \\
\hline
\end{tabular}


Existen otras series clínicas, las que se comparan en la tabla $314,19,20,21$. Aunque tenemos escasas series clínicas recientes, la prevalencia de las causas de HTAS no parece haber variado muy significativamente en el tiempo, excepto por la tasa de prevalencia del hiperaldosteronismo primario, motivado posiblemente por un cambio en su definición y/o en los métodos de estudio (relación concentración de aldosterona plasmática/actividad de renina plasmática) ${ }^{23}$.

Finalmente, parece ser más práctico desde el punto de vista clínico y para el paciente, definir HTAS como aquella HTA que desaparece al erradicar la causa. En consecuencia, se hace necesario para los clínicos analizar los estudios de HTAS considerando las posibilidades ciertas de curación de una HTA.

\section{Referencias}

1. LARAGH J, BRENNERAGH B. Hypertension: pathophysiology, diagnosis, and management. 2nd edition. Lippincott Williams \& Wilkins Inc, 1994.

2. PASSALACQUA W. Hipertensión arterial secundaria. Boletín de Hipertensión 2007.

3. STEWART AB, AHMED R, TRAVILL CM, NEWMAN CG. Coarctation of the aorta life and health 20-44 years after surgical repair. Br Heart J 1993; 69: 65-70.

4. PLOUIN PF, AMAR L, CHATELLIER G. Trends in the prevalence of primary aldosteronism, aldosterone-producing adenomas, and surgically correctable aldosteronedependent hypertension. Nephrol Dial Transplant 2004; 19: 774-7.

5. BONELLI FS, MCKUSICK MA, TEXTOR SC, KOS PB, STANSON AW, JOHNSON CM, et al. Renal artery angioplasty: technical results and clinical outcome in 320 patients. Mayo Clin Proc 1995; 70: 1041-52.

6. WERBEL SS, OBER KP. Pheochromocytoma. Update on diagnosis, localization, and management. Med Clin North Am 1995; 79: 131-53.

7. SLOVUT DP, OLIN JW. Fibromuscular dysplasia. N Engl J Med. 2004; 350: 1862-71.

8. TAGLE R, ACEVEDO M, XU M, POHL M, VIDT D. Use of endovascular stents in atherosclerotic renovascular stenosis: blood pressure and renal function changes in hypertensive patients. J Clin Hypertens (Greenwich) 2007; 9: 608-14.

9. ONUSKO E. Diagnosing secondary hypertension. Am Fam Physician 2003; 67: 67-74.

10. CALHOUN DA. Resistant or difficult-to-treat hypertension. J Clin Hypertens (Greenwich) 2006; 8: 181-6.

11. KIEFER D, PANTUSO T. Panax ginseng. Am Fam Physician 2003; 68:539-42.

12. HALL JE. Pathophysiology of obesity hypertension. Curr Hypertens Rep 2000; 2:139-47.

13. PAPAPIETRO K, DIAZ E, CSENDES A, DÍAZ JC, BRAGHETTO I, BURDILES P, et al. [Effects of gastric bypass on weight, blood glucose, serum lipid levels and arterial blood pressure in obese patients] Rev Med Chil 2005; 133: 511-6.

14. OMURA M, SAITO J, YAMAGUCHI K, KAKUTA Y, NISHIKAWA T. Prospective study on the prevalence of secondary hypertension among hypertensive patients visiting a general outpatient clinic in Japan. Hypertens Res 2004; 27: 193-202.

15. IZZO J and BLACK H. Management of secondary hypertension. Hypertension Primer: The Essentials of High Blood Pressure. 3th Edition. Lippincott Williams \& Wilkins Inc, 2003.

16. KAPLAN NM. Clinical hypertension. 6th Edition. Lippincott Williams \& Wilkins Inc, 1994.

17. MOSSO L, CARVAJAL C, GONZÁLEZ A, BARRAZA A, AVILA F, MONTERO J, et al. Primary aldosteronism and hypertensive disease. Hypertension 2003; 42:161-5.

18. ANDERSON GH JR. BLAKEMAN N. STREETEN DH. The effect of age on prevalence of secondary forms of hypertension in 4429 consecutively referred patients. J Hypertens 1994: 12: 609-15.

19. RUDNICK KV, SACKETT DL, HIRST S, HOLMES C. Hypertension in a family practice. Can Med Assoc J 1977; 117: 492-497.

20. DANIELSON M, DAMMSTRÖM B. The prevalence of secondary and curable hypertension. Acta Med Scand 1981; 209: 451-455.

21. SINCLAIR AM, ISLES CG, BROWN I, CAMERON H, MURRAY GD, ROBERTSON JW. Secondary hypertension in a blood pressure clinic. Arch Intern Med 1987; 147: 1289-1293.

22. PANG TC, BAMBACH C, MONAGHAN JC, SIDHU SB, BUNE A, DELBRIDGE LW, et al. Outcomes of laparoscopic adrenalectomy for hyperaldosteronism. ANZ J Surg 2007; 77: 768-73.

23. MULATERO P, MILAN A, FALLO F, REGOLISTI G, PIZZOLO F, FARDELLA $C$, et al. Comparison of confirmatory tests for the diagnosis of primary aldosteronism. J Clin Endocrinol Metab 2006; 91: 2618-23. 